\title{
OPTMIZATION OF BIO-OPTICAL MODEL PARAMETERS FOR TURBID LAKE WATER QUALITY ESTIMATION USING LANDSAT 8 AND WASI-2D
}

\author{
Manuel, A. ${ }^{1,2}$, Blanco, A.C. ${ }^{1,2}$, Tamondong, A.M. ${ }^{1,2}$, Jalbuena, R. ${ }^{1,2}$, Cabrera, O. ${ }^{3}$, Gege, P. ${ }^{4}$ \\ ${ }^{1}$ Department of Geodetic Engineering, University of the Philippines Diliman \\ ${ }^{2}$ Training Center for Applied Geodesy and Photogrammetry, University of the Philippines Diliman, Quezon City, Philippines \\ e-mail: amimanuel@gmail.com, acblanco@up.edu.ph, amtamondong@up.edu.ph,jalbuena.rj@gmail.com \\ ${ }^{3}$ Institute of Environmental Science and Meteorology, University of the Philippines Diliman \\ email: olive@iesm.upd.edu.ph \\ ${ }^{4}$ German Aerospace Center (DLR), Remote Sensing Technology Institute, 82234 Weßling Germany \\ email: peter.gege@dlr.de
}

KEYWORDS: Remote sensing, inland water, chl-a, bio-optical modeling, inversion

\section{ABSTRACT:}

Laguna Lake, the Philippines' largest freshwater lake, has always been historically, economically, and ecologically significant to the people living near it. However, as it lies at the center of urban development in Metro Manila, it suffers from water quality degradation. Water quality sampling by current field methods is not enough to assess the spatial and temporal variations of water quality in the lake. Regular water quality monitoring is advised, and remote sensing addresses the need for a synchronized and frequent observation and provides an efficient way to obtain bio-optical water quality parameters. Optimization of bio-optical models is done as local parameters change regionally and seasonally, thus requiring calibration. Field spectral measurements and in-situ water quality data taken during simultaneous satellite overpass were used to calibrate the bio-optical modelling tool WASI-2D to get estimates of chlorophyll-a concentration from the corresponding Landsat- 8 images. The initial output values for chlorophyll-a concentration, which ranges from $10-40 \mu \mathrm{g} / \mathrm{L}$, has an RMSE of up to $10 \mu \mathrm{g} / \mathrm{L}$ when compared with in situ data. Further refinements in the initial and constant parameters of the model resulted in an improved chlorophyll-a concentration retrieval from the Landsat- 8 images. The outputs provided a chlorophyll-a concentration range from $5-12 \mu \mathrm{g} / \mathrm{L}$, well within the usual range of measured values in the lake, with an RMSE of $2.28 \mu \mathrm{g} / \mathrm{L}$ compared to in situ data.

\section{INTRODUCTION}

\subsection{Laguna Lake}

Laguna de Bay is the largest freshwater lake in the Philippines and the third in South-East Asia with an area of approximately $900 \mathrm{~km}^{2}$. The lake has been historically, economically, and ecologically significant to the communities surrounding it. Laguna de Bay lies at the center of urban development in Metro Manila, and it suffers from water quality degradation due to intensified exploitation of the its resources along with decades of industrial, household, and toxic pollution (Delos Reyes and Martens, 1994; Tamayo-Zafaralla et al., 2010).

The Laguna Lake Development Authority is a body mandated to address these problems and aim to improve the lake's ecological state along with its 21 major tributary rivers. With the increasing conflict regarding water use, allocation, and preservation of the lake, LLDA is expected to have a comprehensive resource management and monitoring system (Nauta et al, 2003). Implementation of sustainable management strategies should be supported by frequent monitoring (Gray and Shimshack, 2011). However, sampling and analysis used by field-based monitoring programs are labor, cost, and time intensive (Schaeffer et al, 2013). Traditional sampling methods may also produce unevenly distributed sampling points. Data acquired at various times in a day may result to inaccuracies in water quality assessment (Bresciani et al., 2011). In situ measurements can also hardly capture the temporal and spatial variability of large-scale lake phenomena (Dörnhöfer and Oppelt, 2016).

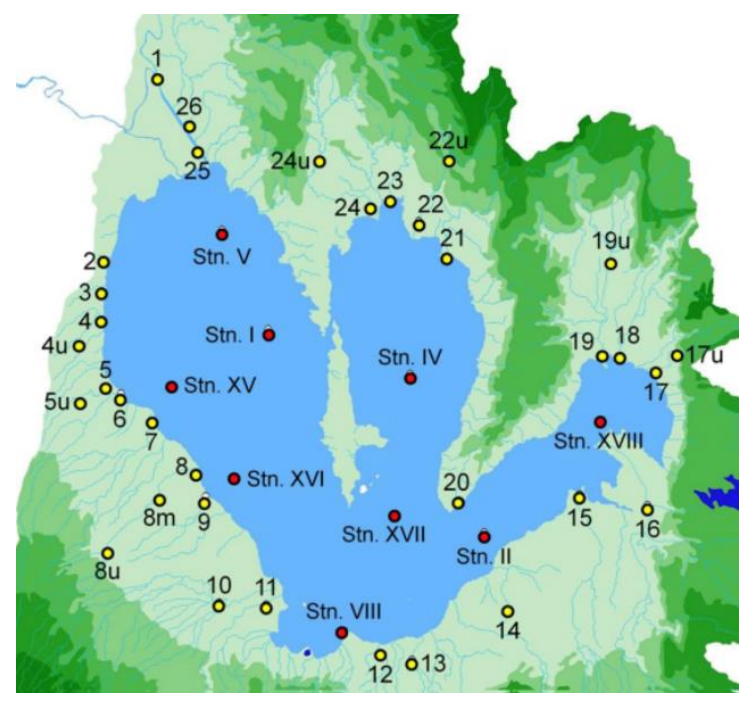

Figure 1. Water quality monitoring stations in Laguna Lake and its tributaries (Source: LLDA)

Water quality monitoring by the Laguna Lake Development Authority are based on 9 sampling stations inside the bay and take measurements once a month (Figure 1). There are instances wherein not all 9 stations are sampled in one day. Even when the sampling is completed within a day, the water quality values are not directly comparable from station to station considering variations within the day. While it is critical to monitor the water quality of an area efficiently and effectively, conventional methods of monitoring produce numerical outputs that are too technical that only few people can understand the implications (Chen, 2009). Also given the extensive area of the lake, the 
limited number of sampling points is not enough to create a visual output that would be easily comprehensible (Khattab and Merkel, 2014). Adding frequent sampling stations would also require considerable efforts in terms of time, labor, and money. These would still be insufficient to detect the changes of the spatial and temporal coverage compared to a detailed map that could be produced from satellite images.

Remote sensing addresses the need for a synchronized and frequent observation, it provides an efficient way to obtain biooptical water quality parameters (i.e. chlorophyll-a, CDOM, SPM) over large areas. Remote Sensing is a very useful measurement tool than can greatly help water management authorities (Chen and Feng, 2018).

Furthermore, free and readily downloadable satellite images from recently launched sensors such as Landsat 8 offer spatial and radiometric resolutions that are suitable for inland water applications (Palmer et al., 2015; Manzo et al., 2015).

\subsection{Bio-Optical Modeling of Inland Waters}

Modelling a relationship between water quality parameters and remote sensing reflectance is a tedious process that requires a deep understanding of hydrologic optics, along with repeated site sampling and simulations that require high computing resources. (IOCCG, 2006) Fortunately, there are already established biooptical models that have been widely used especially for inland water applications. However, parameters for such models can change regionally and seasonally, thus local calibration and fit tuning of the parameters are needed to get good water quality estimate from the satellite images (Gege, 2014).

Due to the presence of multiple constituents at different compositions, remote sensing for monitoring inland quality has been far less common compared to its application to open oceans. The complex interaction among water constituents is often intensified by anthropogenic actions, which creates uncertainty in the models designed for inland waters. Within the same inland aquatic system, different regions can still be dominated by different constituents (Ogasharawa, 2015).

\subsection{Bio-Optical properties and Inherent Optical Properties}

According to Morel (2001), remote sensing of inland waters is based on the optical properties of the constituents in the water. These optical properties are divided into two categories, namely, Apparent Optical Properties (AOPs) that depend on the medium and directional structure of the ambient light field, and Inherent Optical Properties (IOPs), which depend only on the medium itself and independent of the light field (Preisendorfer, 1976). Properties such as absorption (a) and scattering (b) coefficients vary on the composition of the medium or constituents itself (Mobley, 2001).

Absorption properties of water constituents (e.g., algal and nonalgal particles) are the most commonly used parameters since the components in the water can either absorb the light in a specific part of the electromagnetic spectrum and contribute to the color of the water seen by sensors. There are a lot of studies reporting the absorption coefficients from inland waters and most are for sites in USA, China, and South Africa. (Ogashawara, 2017).

Since IOPs vary not only across geographic regions but also within the same site, the complexities are said to be mainly due to the spatio-temporal variability of the water constituents within the same lake. The dominant constituent at the water column may not only change spatially across short distances, but also across seasons and even daily (Gege, 2014). The variability in composition of constituents and associated IOP's in an aquatic system affect the magnitude and shape of the Remote sensing reflectance $\left(\mathrm{R}_{\mathrm{rs}}\right)$. The variations in the $\mathrm{R}_{\mathrm{rs}}$ are related to the variations in IOPs locally at each site (Mobley, 2001).

\subsection{Inversion of Bio-Optical Models}

The forward process in water color optics, is that if the parameters such as particle size, index of refraction of water, distribution of particles, and properties of the dissolved materials are known, the radiance distribution or spectrum of those specific parameters can be simulated by the forward solution of the radiative transfer equation. The inverse modelling, which is used in remote sensing of water bodies, retrieves the Inherent Optical Properties from the radiance and distribution spectrum. From these IOP's, the contribution of constituents such as dissolved matter, particulates from the water spectra can be retrieved (IOCCG, 2006). A simplified diagram of this is shown in Figure 2.

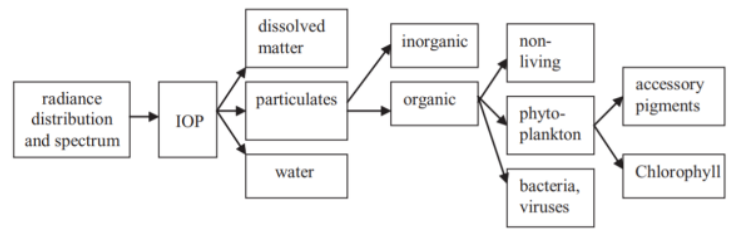

Figure 2. Diagram of inverse radiative transfer elements from Ocean Color Algorithms Working Group Report 5 (IOCCG, 2006)

Inversion of these known relationships determine biogeochemical parameters from the radiance spectrum inversion, however, is inexact and rely on approximations. The inversion is a two-step process: retrieval of IOP's from radiance, then the retrieval of biogeochemical parameters from these IOPs. Both are inexact procedures, which is why spectral inversion must be done with caution and data from field missions (IOCCG, 2006).

Semi-analytical models such as that of Albert and Mobley (2003) and Albert (2004) that are implemented in the Water Color Simulator software rely on the inversion of the Radiative Transfer Equations to establish the relationship among the Remote-sensing reflectance (AOP) and IOPs.

There are existing software that implement these inversion of semi-analytical models, such as the most commonly used for inland waters which is the Water Color Simulator (WASI) software by Peter Gege (2003). The WASI Software tool is developed for analyzing and simulating common types of spectra and allows quantitative analysis of multispectral and hyperspectral images of water bodies. It makes use of the models developed by Albert and Mobley in 2003 and is optimized for inland water applications but can be also applied for oceanic and coastal applications. The two most recent versions WASI 4 and WASI 5 includes the 2D module which applies the spectral inversion models (Figure 3.0) to remote sensing reflectance values in satellite images. This additional component of the software called WASI-2D applies the inverted parameters to atmospherically corrected satellite images and outputs them as estimated water quality concentration maps (Gege, 2014) 


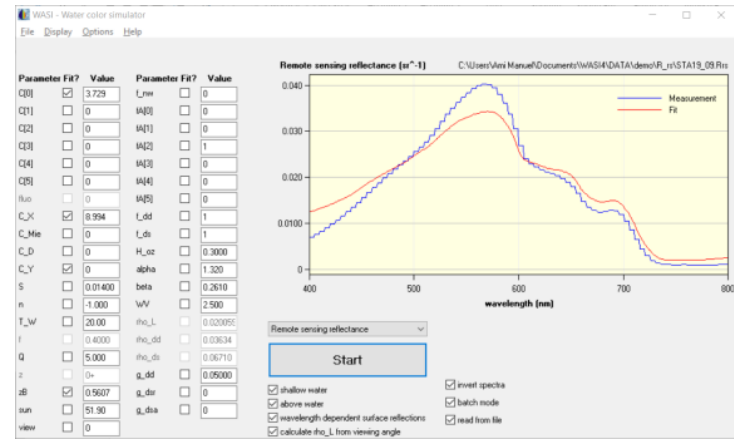

Figure 3. Spectral inversion interface of WASI- Water color simulator

Another example of software implementation of bio-optical models applied to satellite image is the BOMBER software by Giardino, et al. (2012), but it and differs in the implemented biooptical models. The BOMBER software also lacks the individual spectral matching simulation and the option to select specific parameters to fit.

\section{METHODOLOGY}

The bio-optical modelling workflow consists of 2 major parts as shown in Figure 4. The first part is the processing of the field reflectance spectra and assessment of the field acquired IOP spectra. The values outputted from the spectral processing were used as initial inputs and constant parameters when running WASI-2D. The second one consists of the application of the biooptical model inversion on the satellite images incorporating the said values.

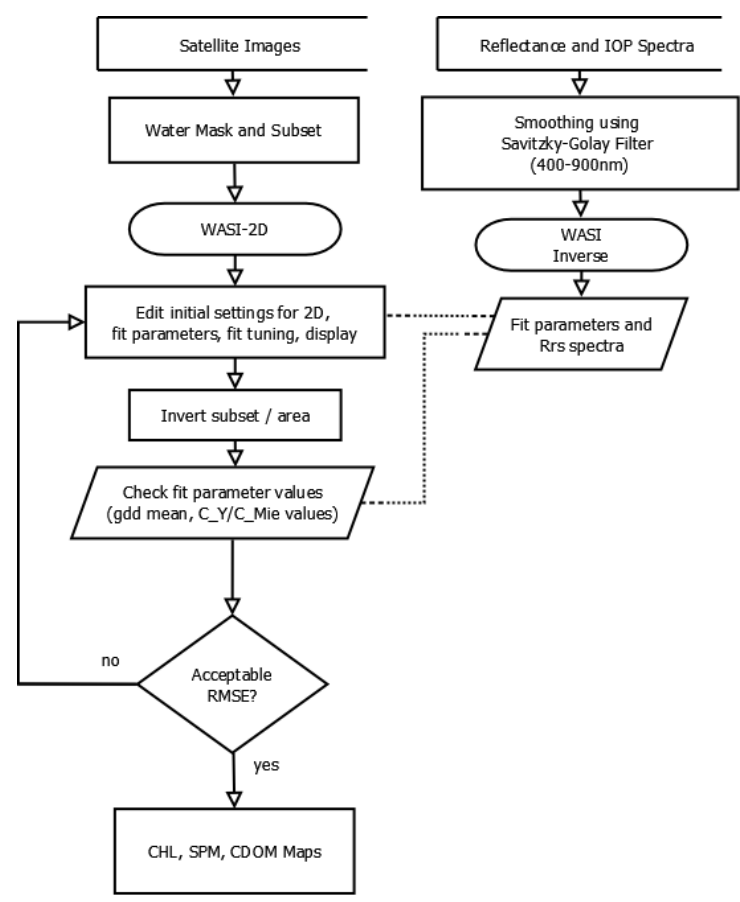

Figure 4. Workflow for the inversion using WASI

\subsection{Data and Field Surveys}

To acquire ground truth values for verification and aid for the inversion process, a 5-day field mission on the Laguna Lake were done for the Dry Season last November 13-17, 2018. The field team acquired data for forty-eight (48) points across the lake. This was done concurrent with different satellite overpass of Landsat 8, Sentinel 2 and Sentinel 3.

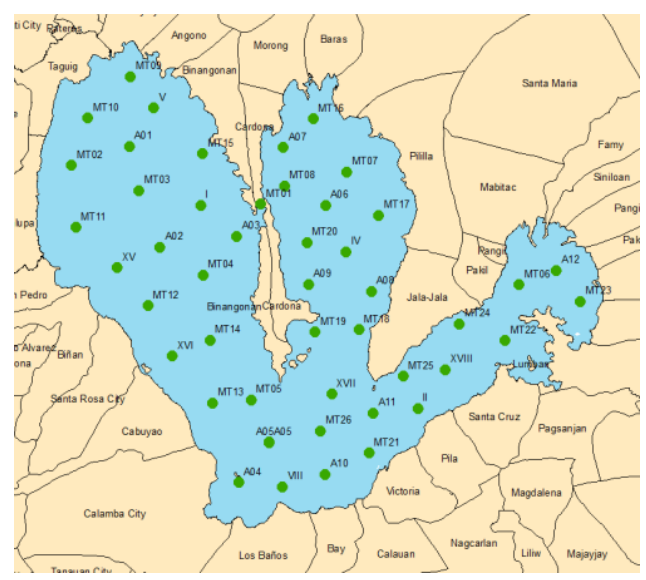

Figure 4. Field Validation Points for Laguna Lake (November 13-17, 2018)

Instruments used in the fieldwork include an Ocean Optics VISNIR Spectrometer (for obtaining reflectance spectra), a Fluoroprobe (submersible fluorometer), and a CLW Infinity Optical Sensor, which measures turbidity, and chl-a. The Fluoroprobe also measures microalgal class concentrations in addition to the total chl-a concentration.

The satellite image used for the bio-optical inversion is a cloud, cloud shadow, and land masked Landsat 8 image. Masking nonwater pixels before running WASI-2D is crucial as spectral inversion may fail. The initial fit parameter input for a pixel takes on the value of the final fit values of the previously processed pixel.

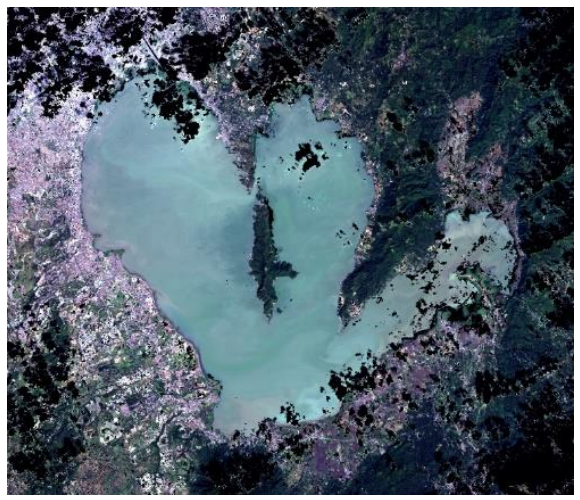

Figure 5. Cloud and cloud shadow masked Landsat 8 image from December 18, 2018

\subsection{Field Spectra and Satellite Image Inversion using WASI}

Since spectral inversion can result in an infinite combination of different fit parameters, some of the constant parameter values must be set in accordance to the properties of the water body. This can be done with the spectral inversion of sample spectral signatures of Laguna Lake waters. Before proceeding with the inversion process with WASI, a spectral smoothing using the Savitzky-Golay smoothing filter was applied to the spectral signature data using a python script (Figure 6). 


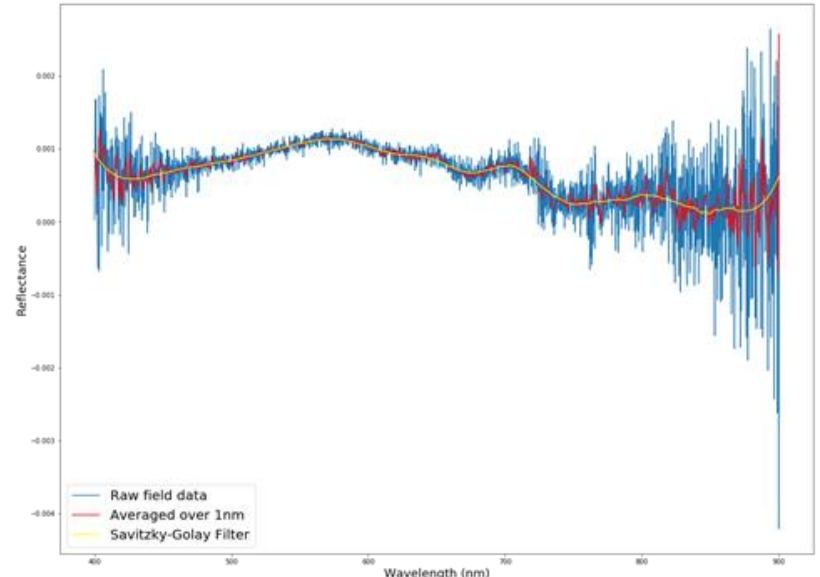

Figure 6. Field sample spectral data smoothing using SavitzkyGolay filter

Initial WASI run gave values and results that do not fall within the field acquired values for chlorophyll. This is due to one spectral shape not necessarily corresponding to a unique set of water quality parameters for the same sun illumination patterns. Therefore, incorporation of a constant value or setting of initial parameters, as well as different fit tuning settings were tested out to improve model performance. In situ Chlorophyll-a measurements was used as a constant to help guide the inversion model that this is the baseline or field acquired value, so it would set the initial simulated spectra closer to the actual field value.
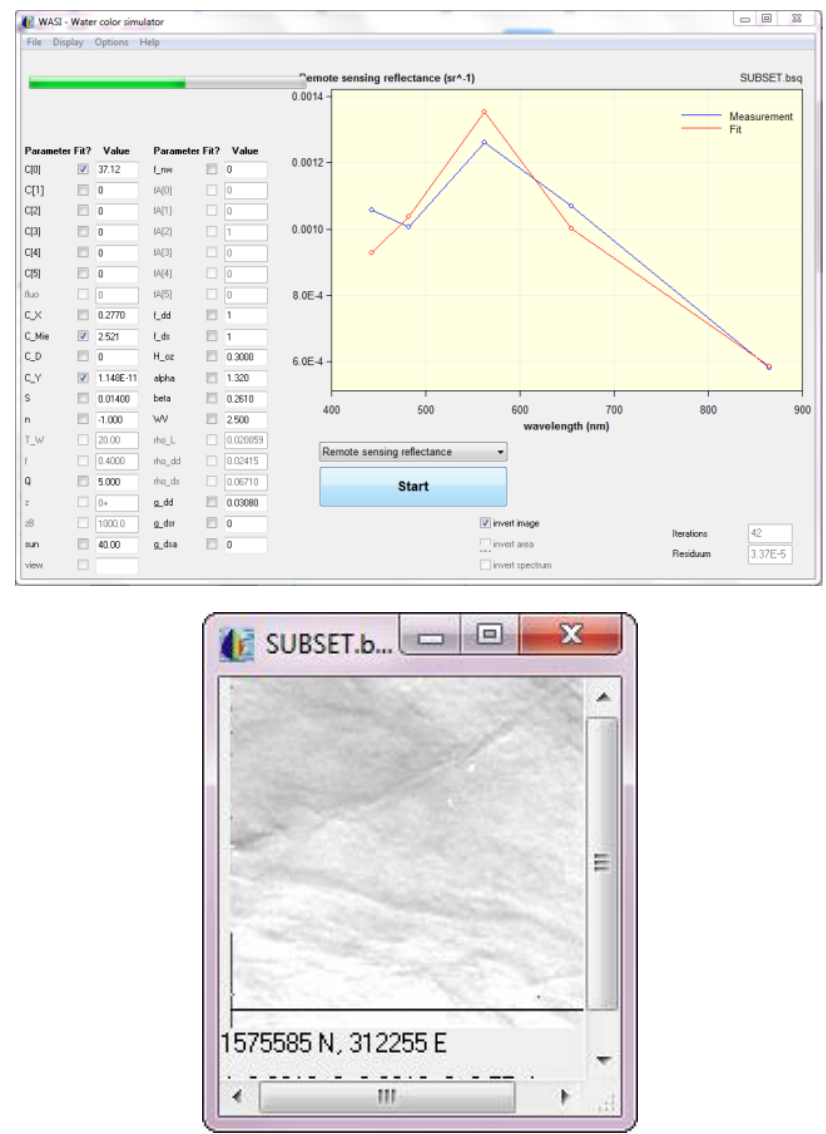

Figure 7. WASI 2-D Interface

Tests for which parameters would be fit and constant as well as setting adequate parameter ranges and initial values were done to get optimal results.

\section{RESULTS AND DISCUSSION}

Out of the 48 points gathered from the field mission, only 27 were useful for ground truth assessment. An inevitable disadvantage of using optical satellite imagery are cloud covers, as the data underneath the clouds will be irretrievable and masked out. The chlorophyll-a concentration output of the best performing run is shown in Figure 8 below.

The first few runs of the WASI software were overapproximating the values up to $40 \mu \mathrm{g} / \mathrm{L}$. These yielded a low $\mathrm{R}^{2}$ value of 0.16 and an RMSE of up to $10 \mu \mathrm{g} / \mathrm{L}$. Aside from the low correspondence to field data, the resulting fit values for CDOM were at 0 . For these initial runs, the contributions for sky radiance specifically the parameter gdd (fraction of sky radiance due to direct solar radiation) were fit and the C_Mie (concentration of non-algal particles of type II) was set at a constant value of 0 . Fitting the gdd correction for sky glint might be affecting the scale of the fit spectra thus affecting the estimation of chlorophyll-a.

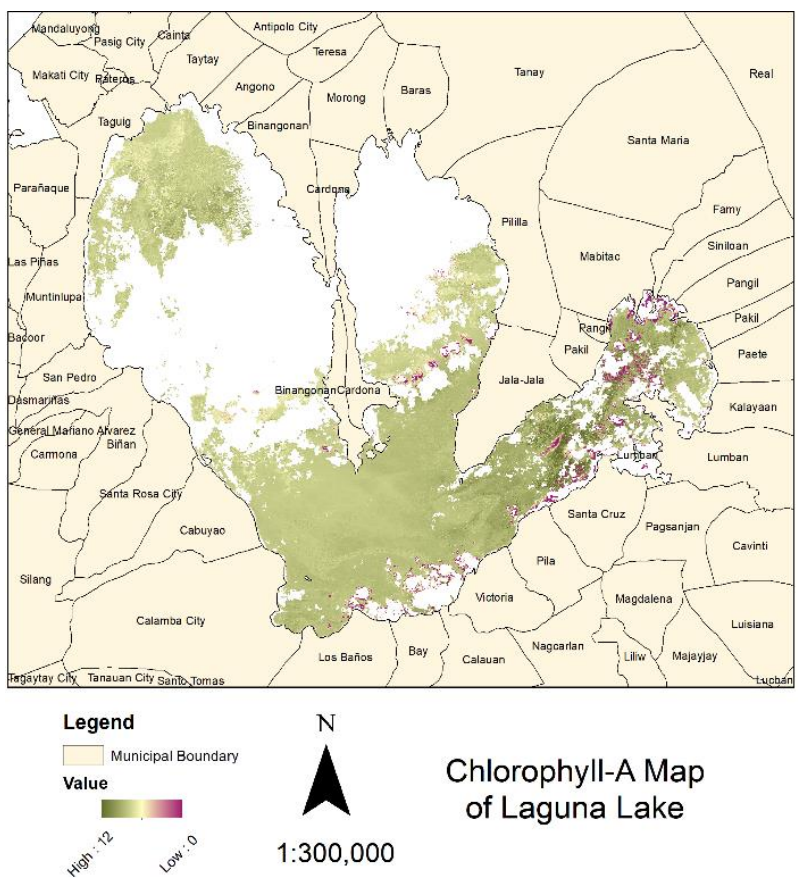

Figure 8. Chlorophyll-a map output from Landsat 8 processing in WASI

Limiting the range of the fit parameter in the program settings and setting the gdd as a constant based on the inverted field spectral values improved the results. Addition of the C_Mie parameter as a fit also resulted in better approximations for chlorophyll-a and gave reasonable values for CDOM. Different runs of the WASI software testing out optimum fit tuning (residuals and maximum iterations) and parameter settings which brought the values within the range of the field monitored values were done. 


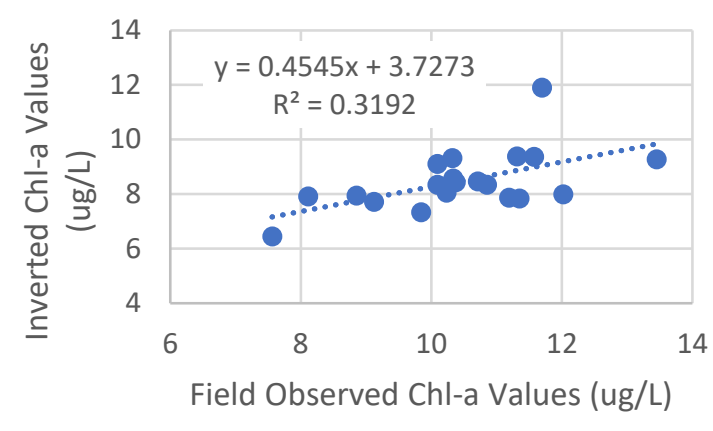

Figure 9. Assessment of Inverted and Field Observed Chlorophyll-a values

The best run yielded an $\mathrm{R}^{2}$ of 0.32 with an RMSE of $2.28 \mu \mathrm{g} / \mathrm{L}$, an improvement to the initial runs. The fitted parameters for the best performing run were chlorophyll-a $(\mathrm{C}[0])$, suspended particulate matter type I (C_X), suspended particulate matter type mie (C_Mie), and colored dissolved organic matter (C_Y). SPM Type 1 (C_X) was tested out as a constant value but it did not yield good approximations compared to when it is fit along with C_Mie. The upper left portion of the output image produced inconsistent values for all the runs. This may be due to the presence of haze or thin cloud cover that were not masked out during pre-processing.

Other parameters such as illumination were kept constant based on the satellite overpass situation and values inverted from field spectral data. Water temperature was also set at a constant of $28.5^{\circ} \mathrm{C}$. Assessment of SPM and CDOM fit performance were not done because of the limitations in the available ground truth data.

\section{CONCLUSION}

Setting up the WASI software incorporating field inverted spectra values to the fit tuning and parameter settings improved the fit performance of the bio-optical models. The inversion of the models on Landsat 8 fitting chlorophyll-a gave an $\mathrm{R}^{2}$ of 0.32 with an RMSE of $2.28 \mu \mathrm{g} / \mathrm{L}$. The outputs of WASI-2D were able to give a visual of the spatio-temporal variation of the chlorophyll-a concentration from a Landsat 8 image.

SPM and CDOM performance show promising results but inconclusive. Further work relating the turbidity field data to SPM and Fluoroprobe outputs to CDOM should be done to assess the other fit parameter outputs of WASI.

\section{ACKNOWLEDGEMENTS}

This study was conducted as a part of the Multi-platform and Cross-sensor Water Quality Monitoring (MCWQM) Project. The Project was implemented by the University of the Philippines Training Center for Applied Geodesy and Photogrammetry (TCAGP), funded by the Department of Science and Technology and the Philippine Council for Industry, Energy, and Emerging Technology Research and Development (PCIEERD). The Project was also done in collaboration with the Laguna Lake Development Authority (LLDA).

Ms. Manuel would also like to acknowledge the Engineering Research and Development for Technology (ERDT) Program and the University of the Philippines Office of International Linkages (UP OIL) for granting her the privilege to present this research in the ISRSE PECORA 21 conference in Baltimore, Maryland, USA.

\section{REFERENCES}

Albert, A., Mobley, C.D. (2003). An analytical model for subsurface irradiance and remote sensing reflectance in deep and shallow case-2 waters. Optics Express, 11 (2003), pp. 2873-2890

Albert, A. (2004). Inversion technique for optical remote sensing in shallow water. Ph.D. thesis, University of Hamburg. http://www.sub.uni-hamburg.de/opus/volltexte/2005/2325

Bresciani, M., Stroppiana, D., Odermatt, D., Morabito G., Giardino, C. (2011). Assessing remotely sensed chlorophyll-a for the implementation of the Water Framework Directive in European perialpine lakes. Science of The Total Environment 409(17):3038-91. doi.org/10.1016/j.scitotenv.2011.05.001.

Chen, S., Fang, L., Zhang, L. and Huang, W. (2009). Remote sensing of turbidity in seawater intrusion reaches of Pearl River Estuary - A case study in Modaomen water way, China. Estuarine, Coastal and Shelf Science, 82(1), pp.119-127.

Chen, X. and Feng, L. (2018). Remote Sensing of Lakes' Water Environment. Comprehensive Remote Sensing, pp.249-277.

Delos Reyes, M. and Martens, R. (1994). Geoecology of Laguna de Bay, Philippines I. Techno-commercial impact on the trophic level structure of the Laguna de Bay aquatic ecosystem 1968-1980. Ecological Modelling, 75-76, pp.497509.

Dörnhöfer, K. and Oppelt, N. (2016). Remote sensing for lake research and monitoring - Recent advances. Ecological Indicators, 64, pp.105-122.

Gege, P. (2004). The water color simulator WASI: an integrating software tool for analysis and simulation of optical in situ spectra. Computers \& Geosciences, 30(5). pp. 523-532.

Gege, P. (2014) WASI-2D: A software tool for regionally optimized analysis of imaging spectrometer data from deep and shallow waters. Computers and Geosciences, (62). pp. 208-215.

Gege, P. (2017) Chapter 2: Radiative Transfer Theory for Inland Waters. Bio-optical Modeling and Remote Sensing for Inland Waters. pp. 25-67.

Giardino, C., Candiani, G., Bresciani, M., Lee, Z., Gagliano, S., Pepe, M. (2012). BOMBER: A tool for estimating water quality and bottom properties from remote sensing images. Computers and Geosciences (45). pp 313-318.

Gray, W. and Shimshack, J. (2011). The Effectiveness of Environmental Monitoring and Enforcement: A Review of the Empirical Evidence. Review of Environmental Economics and Policy, 5(1), pp.3-24.

IOCCG (2006). Remote Sensing of Inherent Optical Properties Fundamentals, Tests of Algorithms, and Applications. Lee, Z.P. (ed.), Reports of the International Ocean-Colour Coordinating Group, No. 5, IOCCG, Dartmouth, Canada.

Khattab, M. and Merkel, B. (2013). Application of Landsat 5 and Landsat 7 images data for water quality mapping in Mosul Dam Lake, Northern Iraq. Arabian Journal of Geosciences, 7(9), pp.3557-3573. 
Manzo, C., Bresciani, M., Giardino, C., Braga, F. and Bassani, C. (2015). Sensitivity analysis of a bio-optical model for Italian lakes focused on Landsat-8, Sentinel-2 and Sentinel-3. European Journal of Remote Sensing, 48(1), pp.17-32.

Mobley, C.D. (2001). Radiative transfer in the ocean. In: Steele, J.H. (Ed.). Encyclopedia of Ocean Sciences. Academic Press Elsevier. London, UK. Pp. 2321-2330

Morel, A., (2001). Bio-optical models. In: Steele, J.H. (Ed.). Encyclopedia of Ocean Sciences. Academic Press Elsevier. London, UK. pp. 317-326

Nauta, T., Bongco, A. and Santos-Borja, A. (2003). Set-up of a decision support system to support sustainable development of the Laguna de Bay, Philippines. Marine Pollution Bulletin, 47(1-6), pp.211-218.

Ogashawara, I., Deepak, R.M., Gitelson, A.A. (2017) Chapter 1: Radiative Transfer Theory for Inland Waters. Bio-optical Modeling and Remote Sensing for Inland Waters. pp. 1-24.

Palmer, S., Kutser, T. and Hunter, P. (2015). Remote sensing of inland waters: Challenges, progress and future directions. Remote Sensing of Environment, 157, pp.1-8.

Preisendorfer, R.W. (1976). Hydrologic Optics (2). U.S. Dept. of Commerce. Washington, DC, USA.

Schaeffer, B., Schaeffer, K., Keith, D., Lunetta, R., Conmy, R. and Gould, R. (2013). Barriers to adopting satellite remote sensing for water quality management. International Journal of Remote Sensing, 34(21), pp.7534-7544.

Tamayo-Zafaralla, M., Santos, R., Orozco, R. and Elegado, G. (2002). The ecological status of Lake Laguna de Bay, Philippines. Aquatic Ecosystem Health \& Management, 5(2), pp.127-138. 